\title{
Zur Entwicklung quartärer Hohlformen in Franken
}

\author{
Von Arno Semmel, Frankfurt a. M., und Gerhard Stäblein, Würzburg \\ Mit 6 Abbildungen
}

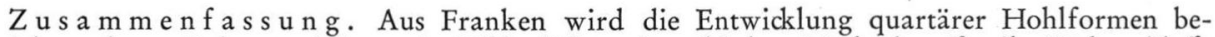
schrieben, deren Rekonstruktion mit Hilfe lößstratigraphischer Methoden (fossile Böden, Tuffbänder, Umlagerungszonen etc.) möglich ist. Bei vielen Formen zeigt sich, daß sie bereits größere Vorläuferformen präwürmzeitlichen Alters hatten. Die Entwicklung während des Würms läßt sich an manchen Beispielen in besonders instruktiver Weise verfolgen.

$\mathrm{Zu}$ Beginn des Würms, im unteren Mittelwürm und im unteren Jungwürm dominierte zeitweise die Abtragung und Verlagerung. Im oberen Mittelwürm sowie im oberen Jungwürm herrschte äolische Lößsedimentation vor. Diese Ergebnisse stimmen gut mit den bereits aus anderen mitteleuropäischen Lößgebieten bekannten Befunden überein.

Mit dem Trockental-System von Helmstadt wird die Entwicklung von Hohlformen beschrieben, deren Anlage bis in das ältere Pleistozän zurückreicht.

$\mathrm{Su} \mathrm{m} \mathrm{m}$ a r y. The development of quaternary hollow forms in Franconia (south Germany) is described. Its reconstruction is possible by methods of loess stratigraphy (fossil soils, tuff stripes, resedimentation layers, etc.). Many forms turn out to have had already greater precedent forms of pre-Würm age. During the Würm period the development can be very instructively traced back by several examples.

At the beginning of the Würm period, in the lower middle Würm and in the lower late Würm erosion and displacements predominated at times. Eolian loess sedimentation prevailed in the upper middle Würm and in the upper late Würm. These results coincide well with the observations of other already well known central European loess areas.

The development of hollow forms is described by the dry valley system of Helmstadt, the origin of which goes back to the earlier Pleistocene.

\section{Einleitung}

In den letzten Jahren sind in den deutschen Mittelgebirgen Versuche unternommen worden, die Entwicklung von Hängen und Hohlformen, die die Hänge gliedern, im einzelnen zu erfassen (vgl. z. B. BARTELS \& RoHdEnburg 1968; Fränzle 1969; RoHdenburg 1965, 1968; Semmel 1961, 1968). Die hier vorgelegten Befunde sollen die bisher aus anderen Gebieten bekannten Ergebnisse mit Beispielen aus Franken (Abb. 1) ergänzen und erweitern. Zunächst werden zwei Beispiele aus der Umgebung von Kitzingen a. M. erörtert, die schon früher von BRUNNACKER $(1956,1959)$ lößstratigraphisch untersucht worden sind. In beiden Profilen lassen sich die einzelnen Formungsstadien von Dellen in selten zu findender Anschaulichkeit beobachten. Die weiteren Beispiele zeigen ebenfalls, daß auch in Franken die schon aus anderen Gebieten berichteten Einzelphasen des Formungsverlaufs von entsprechenden Formen zu finden sind. Darüber hinaus wird mit dem Helmstädter Trockentalsystem aber ein Objekt diskutiert, dessen Genese besonders weit in die Vergangenheit zu verfolgen ist. Ahnliches gilt auch für das aus der Nähe von Bamberg erwähnte Beispiel.

Mit Ausnahme des letztgenannten, nur kurz erörterten Aufschlusses sind es Profile, in denen der Löß dominiert. Die intensive, vorwiegend auf paläopedologischer Grundlage fußende Lößstratigraphie-Forschung der letzten zwanzig Jahre hat dazu geführt, daß vor allem für das Jungpleistozän ein Kenntnisstand erreicht ist, der bisher wohl kaum in anderen erdgeschichtlichen Bereichen erzielt werden konnte. Hiermit wurden aber auch gleichzeitig die Voraussetzungen für eine diffizile Rekonstruktion der Reliefentwicklung in Lößgebieten gegeben. In anderen Substraten gelingt ähnliches zur Zeit noch nicht mit 
vergleichbarer Genauigkeit. Die Gründe dafür wurden bereits an anderer Stelle erörtert (Semmel 1968, 59). Der Löß spricht besonders gut auf die Verwitterung an. Es genügen schon geringfügige Unterbrechungen oder Veränderungen während der Sedimentation und dem oft damit verbundenen Wirksamwerden von Bodenbildungsvorgängen, um deutliche Spuren im Löß zu hinterlassen. Die anschließend wieder einsetzende Lößakkumulation deckte solche Spuren meist sehr sorgfältig ein und sorgte für ihre Erhaltung bis in die heutige Zeit.

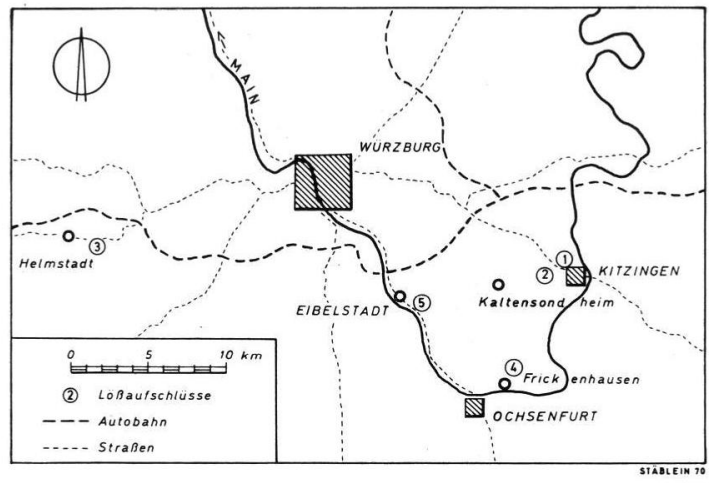

Abb. 1. Lage der untersuchten Aufschlüsse. $1=$ Lößgrube am Kitzinger Friedhof; $2=$ Lößgrube an der Straße Kitzingen-Kaltensondheim; $3=$ Lößgrube östlich Helmstadt; $4=$ Delle bei Frikkenhausen; $5=$ Aufgefüllte Delle bei Eibelstadt.

Nach dem Ende der Lößsedimentation im ausgehenden Pleistozän bildete sich ein intensiver Boden, der die Form nachzeichnet, die am Ende des Pleistozäns bestand. Dort, wo dieser Boden nicht in seinem natürlichen Reifezustand (Klimaxstadium) erhalten ist oder unter anderen Sedimenten liegt, hat auch in holozäner Zeit eine deutliche Reliefveränderung stattgefunden. Somit kann auch über die Formenentwicklung im Holozän etwas ausgesagt werden, wenn man bodenkundliche Befunde heranzieht. Die vorliegende Arbeit versucht also einmal mehr zu zeigen, wie nützlich die Anwendung bodenkundlicher Methoden in der Geographie ist.

\section{Die Entwicklungsstadien von Dellen in der Umgebung von Kitzingen a. M.}

\subsection{Lößgrube an der Straße Kitzingen-Kaltensondheim}

Etwa $2 \mathrm{~km}$ westlich des Stadtzentrums von Kitzingen liegt an der Straße nach Kaltensondheim eine Lößgrube der Ziegelei PAvel \& BeCkeR, die bereits von Brunnacker (1959) lößstratigraphisch untersucht wurde. Der Aufschluß schneidet eine Delle an, die von Norden in das Tal des Eherieder Baches einmündet. Das Tal zeichnet sich in diesem Bereich durch einen flacheren süd- und einen steileren nordexponierten Hang aus. Es besitzt einen 30-40 m breiten Talboden, auf dem aus der Delle ein kleiner Schwemmfächer ausläuft. An der talaufwärts gelegenen Grenze des Schwemmfächers sind in der Aue ca. $1 \mathrm{~m}$ Auenlehm und darunter 0,5 bis 1,5 $\mathrm{m}$ Kies erbohrt worden, der den anstehenden Keuper bedeckt. Außerhalb der Aue steigt nach Norden die Oberfläche des anstehenden Lettenkeupers um ca. $1,5 \mathrm{~m}$ an. Darüber liegt ein 0,5 bis $1 \mathrm{~m}$ mächtiger Schwemmschutt aus Keupermaterial, der die Basis einer Lößdecke bildet (Abb. 2). Die Lößbasis befindet sich also fast im Niveau des Auenbodens. Der Eherieder Bach hat sich seit der Ablagerung des Schwemmschuttes, der die Lößbasis bildet, nur ganz geringfügig eingetieft. In ähnlicher Weise ist auch die Delle an keiner Stelle durch den basalen Schwemmschutt hindurch ein- 
getieft worden, obwohl - wie noch zu zeigen sein wird - der Schwemmschutt mindestens in die vorletzte Kaltzeit gehört und die Entwicklung der Delle von damals bis heute andauert.
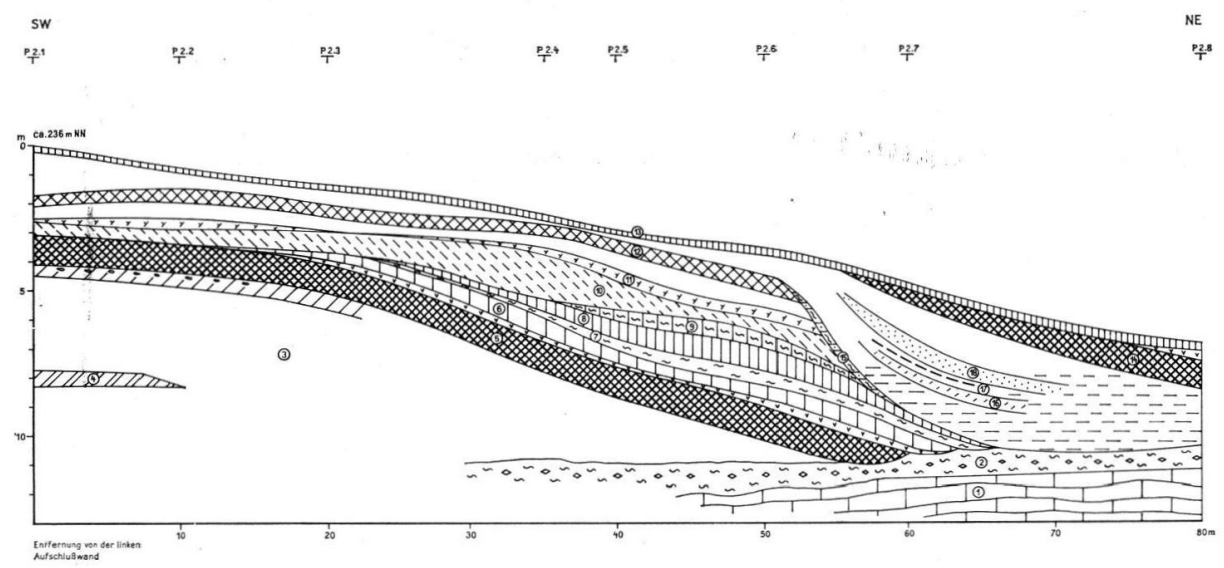

Abb. 2. Lößgrube an der Straße Kitzingen-Kaltensondheim (R 358150 / H 551162)

$1=$ anstehender Keuper; $2=$ Schwemmschutt; $3=$ Riß-Löß; $4=$ „Altriß/Jungriß-Boden“; $5=$ Parabraunerde der letzten Warmzeit; $6=$ Altwürm-Humuszone (ältere); $7=$ KeuperFließerde; $8=$ Altwürm-Humuszone (jüngere); 9 und $10=$ umgelagertes Boden- und LößMaterial; $11=$ Niedereschbacher Zone (?); $12=$ Lohner Boden („Brauner Verwitterungshorizont $\left.^{\prime \prime}\right) ; 13=$ Pararendzina; $14=$ Parabraunerde; $15=$ umgelagerter Lohner Boden; $16=\mathrm{Naß}$ boden $\mathrm{E}_{2} ; 17=$ Eltviller Tuff; $18=$ Naßboden $\mathrm{E}_{4}$. P 2.1-P $2.8=$ aufgenommene Einzelprofile.

Úber dem Schwemmschutt (Nr. 2 auf Abb. 2) wurde zunächst die Lößdecke (3) sedimentiert, die stellenweise mindestens $7 \mathrm{~m}$ mächtig war. Sie enthält eine schwächere Bodenbildung (4), den „Altriß/Jungriß-Boden“ BrunnaCKers (1959, 140). Auf dem Löß bildete sich eine Parabraunerde, die von Brunnacker in die letzte Warmzeit eingestuft wird. Wir fanden keinen dagegen sprechenden Befund. Die Parabraunerde (5) taucht im nordöstlichen Teil des Aufschlusses bis auf den Schwemmschutt ab. Der Verlauf des Bodens gibt den Zustand der einen Dellenhälfte am Ende der letzten Warmzeit wieder. Der kräftig ausgeprägte Klimaxboden zeigt weitgehende Formungsruhe zu dieser Zeit an.

$\mathrm{Zu}$ Beginn der letzten Kaltzeit setzen erste Verlagerungen ein, die sich darin äußern, $\mathrm{daß}$ Material des $\mathrm{fA}_{\mathrm{l}}$-Horizontes der Parabraunerde als Fließerde nach Osten in den Schwemmschutt einschneidet. Die Argumente von Brunnacker (1959, 134 f.) für interglaziale Bodenverlagerungen sind u. E. nicht zweifelsfrei. Auch Rohdenburg \& Meyer (1966, 30) bringen hiergegen bereits Argumente. Neben den dort angeführten sei noch erwähnt, daß aus der Mächtigkeit eines $\mathrm{B}_{\mathrm{t}}$-Horizontes keine Rückschlüsse auf die Mächtigkeit des dazugehörenden $\mathrm{A}_{1}$-Horizontes gezogen werden können, denn der Tongehalt des $\mathrm{B}_{\mathrm{t}}$-Horizontes steht $\mathrm{nicht} \mathrm{nur}$, oft nachweislich überhaupt nicht mit einer Tonzufuhr aus dem $A_{1}$-Horizont in Verbindung (Semmel \& Plass 1965). Daß die betreffende Verlagerung kaltzeitlichen Charakter hatte, läßt sich zwar in den Kitzinger Profilen nicht sicher nachweisen, aber in Helmstedt (vgl. S. 31) ist der $\mathrm{A}_{\mathrm{l}}$-Horizont in Eiskeile eingesunken, die an der Oberkante des $\mathrm{B}_{\mathrm{t}}$-Horizontes ansetzen und somit die Eigenständigkeit des hangenden Substrates beweisen (vgl. hierzu Semmel 1968, 25 ff.). Die meisten der sogenannten $\mathrm{A}_{1}$-Horizonte solcher Böden sind Fließerden, die bereits frisch sedimentiertes Lößmaterial enthalten, also kaltzeitlich beeinflußt wurden. Deshalb überrascht auch nicht das Fehlen von $\mathrm{B}_{\mathrm{t}}$-Merkmalen im Keuper-Schwemmschutt unter dem " $\mathrm{A}_{\mathrm{l}}$-Horizont“. Solche Tonverlagerungsanzeichen bilden sich indessen ohnehin häufig $\mathrm{nicht}$ in ähnlichen Gesteinen. Näheres zu diesem Fragenkomplex findet sich bei Semmel (1968, 67). 
Nach dieser Fließerdebildung wurde der umgelagerte $A_{1}$-Horizont an manchen Stellen abgetragen. Anschließend erfolgte eine Eindeckung mit Lößmaterial, auf dem sich dann eine Schwarzerde (6) entwickelte. Die mit ihr verbundene Humifizierung reicht im Dellentiefsten bis in den $\mathrm{fB}_{\mathrm{t}}$-Horizont hinein. Nach dieser Phase relativer Formungsruhe überwanderte eine Keuper-Fließerde (7) die Schwarzerde und kappte sie im Dellentiefsten. Die Fließerde wurde wiederum von Lößmaterial überlagert, auf dem abermals eine Schwarzerde (8) entstand. Nach den Beobachtungen von BRUNNACKER (1959, 138 ff.) lag darüber noch einmal eine Keuper-Fließerde und eine hangende Schwarzerde. Heute wird die Schwarzerde (8) von einer Diskordanz geschnitten, die eine Abtragungsphase anzeigt, welche ihre stärkste Wirkung - soweit heute erkennbar - auf dem Dellenhang im westlichen Teil des Aufschlusses hatte. Diese Abtragungsphase muß, da sie den SchwarzerdeKomplex kappt, der in das Altwürm i. S. von Schönhals et al. (1964) gehört, eben jünger als Altwürm sein.

Auf der Diskordanz liegt verlagertes Lößmaterial (9) und (10), in dem Schwarzerdeund $\mathrm{B}_{\mathrm{t}}$-Reste enthalten sind. Es hat einen hangenden Streifen (11) mit deutlicher Verbraunung. Vielleicht entspricht dieser Bereich der "Niedereschbacher Zone“ (Semmel 1969) einer Verwitterungs- und Umlagerungsperiode im Mittelwürm. Anschließend wurde dann äolischer Löß sedimentiert, aus dem der „Lohner Boden“ (SснӧNHALs et al. 1964) oder der „Braune Verwitterungshorizont" Brunnackers (1959) hervorging, der das Mittelwürm abschließt.

Der über dem Lohner Boden (12) liegende Löß trägt heute im westlichen Teil der Grube eine Pararendzina (13). Die ursprünglich entwickelte Parabraunerde ist durch junge Bodenerosion abgetragen worden. Nur im Dellentiefsten blieb sie in ihrer natürlichen Mächtigkeit erhalten. Hier fehlt indessen der Lohner Boden. Er ist einer Ausräumungsphase zum Opfer gefallen, die in das frühe Jungwürm gehört. Während dieser Zeit entstand eine neue Hohlform, die durch den älteren Würmlöß hindurch bis in den KeuperSchwemmschutt eingetieft wurde. Sie ist mit Schwemm- und Fließlöß ausgefüllt worden. Auf dem westlichen Hang dieser Delle liegt basal Material (15), das vorzugsweise aus dem Lohner Boden stammt und teilweise typische Eigenschaften dieses Bodens trotz der Umlagerung besitzt. Zum Ende des Jungwürms überwog hier die Ablagerung von äolischem Löß mit den Naßböden $\mathrm{E}_{2}$ und $\mathrm{E}_{4}$ sowie dem dazwischen eingeschalteten Eltviller (Kärlicher) Tuff (16-18) (Semmel 1967). Außerhalb der Jungwürm-Delle fehlen diese Horizonte ebenso wie im Dellentiefsten. Dort herrschten auch zu dieser Zeit noch Verspülung und Bodenfließen vor.

Auf der östlichen Dellenseite fehlt der Löß. Dort reicht der Lettenkeuper bis zur Oberfläche. Obwohl hier während des gesamten Jungpleistozäns die Abtragung dominierte, wird zu Beginn des Holozäns - in Analogie zu anderen Profilen - ein Solifluktionsschutt gelegen haben. Aus diesem entwickelte sich im Holozän zunächst eine Braunerde. Die Verbraunung griff teilweise bis in den Keuper. Solche Stellen sind heute noch vorhanden. Der Solifluktionsschutt und mit ihm der größte Teil der Braunerde sind jedoch von der Bodenerosion, die durch die Beackerung ausgelöst wurde, abgetragen worden.

Zusammenfassend gesehen hat diese Hohlform also folgende wesentliche Formungsstadien aufzuweisen:

1. Es bestand eine Hohlform als Vorläuferform, deren Ausdehnung nicht genau bekannt ist, die aber bereits bis zum heutigen Auenniveau und damit auch tiefer als alle anderen nachfolgenden Stadien eingeschnitten war. Diese Vorläuferform entstand vor der letzten Warmzeit.

2. Die Hohlform wurde noch vor der letzten Warmzeit im westlichen Bereich mit Löß aufgefüllt. Am Ende der letzten Warmzeit bestand eine Form, die gewisse Ahnlichkeit mit der heutigen Oberflächenform aufweist. 
3. Zu Beginn der letzten Kaltzeit fand im Dellentiefsten mehrfach eine schwache Ausräumung und gleichzeitig eine geringe Verschiebung der Tiefenlinie nach Nordost statt. Im ganzen überwog aber die Auffüllung in der Delle.

4. Zu Beginn des Mittelwürms macht sich eine stärkere Abtragungsphase bemerkbar, die auf dem westlichsten Hangteil die älteren Böden kappt. Ob sie sich im Dellentiefsten auswirkte, ist nicht festzustellen. Anschließend dominierte wieder die Auffüllung. $\mathrm{Da}$ jedoch in diesem Profil der Gräselberger Boden (ScHÖNHALs et al. 1964) des Mittelwürms fehlt, muß noch später eine möglicherweise nur geringfügige Ausräumung stattgefunden haben.

5. Im älteren Jungwürm läßt sich wieder eine Abtragungsphase erkennen. Ihre Wirkung beschränkt sich auf den Bereich des Dellentiefsten, weist dort aber Eintiefungsbeträge auf, die sonst während des Würms im gesamten Dellenbereich nicht erreicht wurden. Gleichzeitig findet eine weitere Verlegung des Dellentiefsten nach NE und wahrscheinlich eine Verstärkung der Asymmetrie statt.

6. Die nachkaltzeitliche Phase der Formungsruhe wird durch die im Gefolge der Beackerung aufkommende Bodenerosion abgebrochen. Die Klimax-Parabraunerde im Lößbereich wird außerhalb des Dellentiefsten erodiert. Auch die Braunerde im Keuperbereich unterliegt dem gleichen Vorgang.

Dadurch verflacht die Ausgangsform. Auf Grund der bodenkundlichen Befunde dürften diese holozänen Abtragungsbeträge größtenteils kaum 1,20 m überschreiten. Im Keuperbereich findet man nämlich noch Verbraunungsreste, im Lößbereich zeigen stellenweise noch Lößkindl den ungefähren Verlauf des $\mathrm{C}_{\mathrm{c}}$-Horizontes der ehemaligen Parabraunerde an.

\subsection{Lößgrube am Kitzinger Friedhof}

Als weiteres Beispiel einer differenzierten Hohlformenentwicklung sei die dellenähnliche Form am Kitzinger Friedhof angeführt (ehemalige Ziegeleigrube Korbacher). Der dortige Aufschluß läßt sehr gut die Genese des westlichen Hanges und des Dellenzentrums erkennen. Das Profil wurde ebenfalls von Brunnacker (1956) lößstratigraphisch bearbeitet.

Auch hier ist klar ersichtlich, daß die Delle eine Vorläuferform hatte, die tiefer als alle späteren Formungsstadien in den anstehenden Keuper eingeschnitten wurde. Diese Vorläuferform kappt im Westen des Aufschlusses (auf Abb. 3 nicht dargestellt) einen fluvialen Schotterkörper, der 1-2 m über dem Grubenboden liegt. Durch Bohrungen kann eine Mindesteintiefung der Altform bis 3,15 m unter dem Grubenboden nachgewiesen werden. Der tiefste Bereich lag wahrscheinlich noch weiter im Westen. Die Füllung der Hohlform beginnt mit einem aus Keupermaterial bestehenden Schutt (2 auf Abb. 3), der dem „Basisschutt“ (Semmel 1968, 63) entsprechen dürfte. Es folgt ein lößlehmhaltiger Keuperschutt (3), der als "Mittelschutt“ (SEMmel 1968, 63) zu bezeichnen wäre. Durch diesen Schutt (3) wird die alte Delle anscheinend weitgehend aufgefüllt. In den Schutt wurde eine neue Hohlform eingetieft und anschließend mit Löß (4) aufgefüllt. Wie die Reliefentwicklung hierbei im einzelnen verlaufen ist, kann nicht mehr sicher rekonstruiert werden. Als sich der letztwarmzeitliche Boden (5) bildete, bestand jedenfalls wiederum eine Delle.

Der Boden wurde in der beginnenden Würm-Kaltzeit im oberen Hangbereich und im Dellentiefsten erodiert. Nach der Bildung der Altwürm-Schwarzerden (6), die auch „Humuszonen" genannt werden, findet ebenfalls Abtragung statt. Im tieferen Teil wurde später humoser, löß- und keuperhaltiger Schutt (7) sedimentiert. Bemerkenswert ist, daß im östlichen Teil des tieferen Dellenbereiches die älteren Böden vor der Ablagerung dieses Schutts völlig ausgeräumt worden sind. Die jüngere Lößdecke (8) wird von der mit Boden- 
bildung und Umlagerung verbundenen Niedereschbacher Zone (9) abgeschlossen. Diese und auch der auf der hangenden Lößlage (10) entstandene "Gräselberger Boden" (11) sind im höheren Hangbereich gekappt. Der „Lohner Boden“(12), der das Mittelwürm abschließt, blieb dagegen dort weitgehend erhalten.

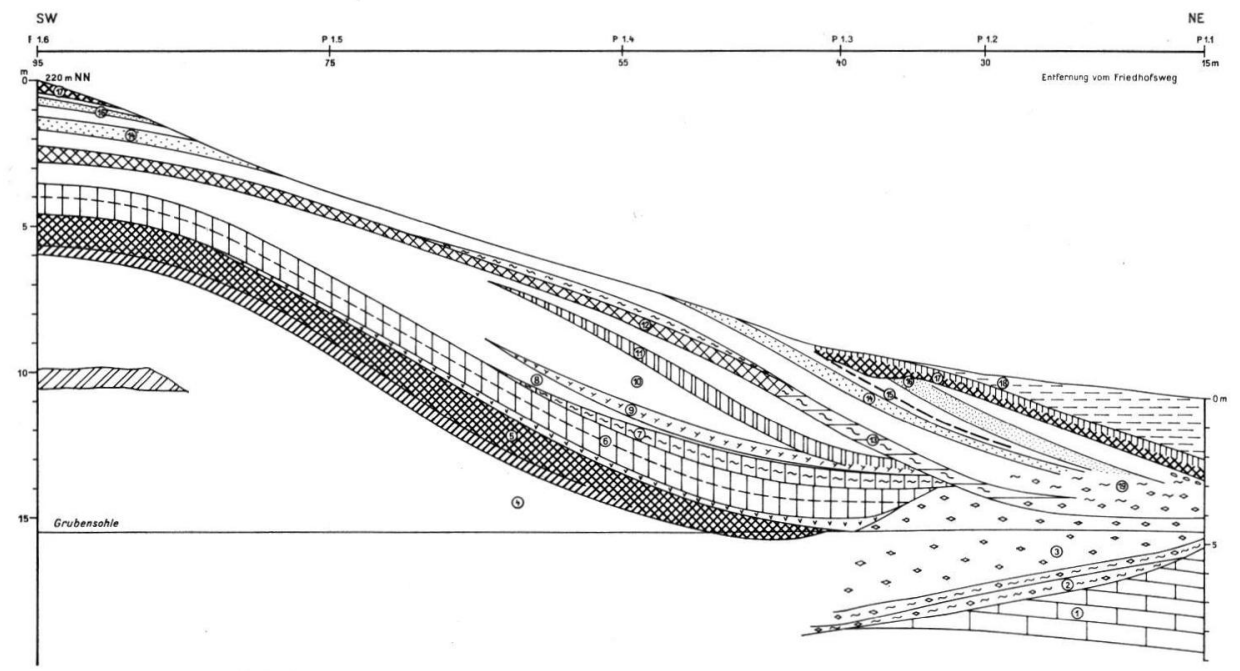

Abb. 3. Lößgrube am Kitzinger Friedhof (R 358287 / H 551266)

$1=$ anstehender Keuper; $2=$ Keuper-Schutt; $3=$ lößhaltiger Keuper-Schutt; $4=$ Rißlöß mit eingeschaltetem Boden; $5=$ Parabraunerde der letzten Warmzeit; $6=$ zweigeteilte AltwürmHumuszone; $7=$ lößlehmhaltiger Keuper-Schutt; $8=$ Mittelwürm-Löß (älterer); $9=$ Niedereschbacher Zone; 10 = Mittelwürm-Löß (jüngerer); 11 = Gräselberger Boden; 12 = Lohner Boden;' $13=$ Lohner Boden, verlagert; $14=$ Naßboden $\mathrm{E}_{2} ; 15=$ Eltviller Tuff; $16=$ Naßboden $\mathrm{E}_{4}$; $17=$ Parabraunerde; $18=$ Kolluvium; $19=$ lößlehmhaltiger Schwemmschutt. P $1.1-\mathrm{P} 1.6=$ aufgenommene Einzelprofile.

Das Jungwürm beginnt wiederum mit einer Abtragungsphase, die sich nur im tiefsten Teil der Delle deutlich auswirkt, und zwar wird hier der Lohner Boden stark solifluidal verlagert (13) und gleichzeitig der Dellenboden eingetieft und nach NE verlegt. Im mittleren und oberen Jungwürm lagert sich vorzugsweise äolischer Löß ab, der die Naßböden $\mathrm{E}_{2}$ und $\mathrm{E}_{4}$ sowie stellenweise den Eltviller Tuff (14-16) enthält. Der $\mathrm{E}_{4}$-Boden ist besonders kräftig im tieferen Dellenteil entwickelt. Im Dellentiefsten selbst wurden zur Bildungszeit des $\mathrm{E}_{4}$-Bodens noch vorwiegend Schwemmsedimente abgelagert. Die Mächtigkeit des noch jüngeren äolischen Lösses beträgt hier nur etwas mehr als $1 \mathrm{~m}$; auch die Mächtigkeit des Schwemmschuttes ist sehr gering. In diesem Bereich dominierte die Abtragung also fast bis zum Ende des Jungwürms.

Die nach Abschluß der Lößsedimentation entstandene Parabraunerde (17) ist im Dellentiefsten stark humos. Sie wurde hier durch Ackerkolluvium (18) überdeckt. Hangaufwärts ist sie auf den steileren Partien völlig erodiert.

Insgesamt gesehen sind die Parallelen zu dem Profil an der Straße nach Kaltensondheim unverkennbar. Die dort ermittelten morphologisch bedeutsamen Abtragungs- und Akkumulationsphasen kommen auch hier vor und die Wanderung der Delle nach NE ist ebenfalls unbestreitbar. Damit bestätigen sich aber auch die in Hessen und Südniedersachsen gewonnenen Befunde. Rohdenburg (1968, 45 ff.) teilt außerdem ähnliche Ergebnisse aus der Umgebung von Heilbronn mit. Nur selten sind bisher aber Beispiele bekannt geworden, in denen die Einzelstadien so klar zu rekonstruieren waren. Das gilt vor allem für die schrittweise Verlegung des Dellentiefsten nach NE und das mehrfach zu beobach- 
tende Zurückbleiben der jüngeren Eintiefung gegenüber älteren Stadien. Darin zeigt sich besonders gute Übereinstimmung mit den Verhältnissen im Rhein-Main-Gebiet (SEMmEL 1968, 116). Allerdings ist dort die Entwicklung in der Regel nicht so beispielhaft zu verfolgen wie an den beiden Formen aus der Umgebung von Kitzingen.

\subsection{Delle bei Frickenhausen}

Daß nicht in jedem Falle die Vorläuferform tiefer eingeschnitten war, zeigt eine Delle, die ca. $8 \mathrm{~km}$ SSW von Kitzingen liegt. Dort sind durch den Bau der Aussiedlerhöfe nordwestlich der Markgrafenhöfe bei Frickenhausen die auf Abb. 4 wiedergegebenen Dellenquerschnitte freigelegt gewesen.

Auffällig ist zunächst die deutliche Asymmetrie, die ja auch bei dem zuerst beschriebenen Beispiel vorhanden war. Die Vorläuferform wird im westlichen Teil gut durch die Grenze des Keuperschuttes (2 auf Abb. 4) zum Löß (3) markiert. Dieser Grenze folgt ungefähr auch noch der Rest des letztwarmzeitlichen Bodens (4) und eine Altwürm-Humuszone (5). Die darüber liegende Diskordanz greift im Dellentiefsten durch den gesamten älteren Löß in den Keuper hinein. Da im basalen Bereich der Füllung dieses Dellenteiles nur der $\mathrm{E}_{2}$-Naßboden (6) ausgebildet ist, kann diese Diskordanz mit großer Wahrscheinlichkeit in das frühe Jungwürm eingeordnet werden. Zu dieser Zeit erreichte also die Delle ihre größte Austiefung. Gleichzeitig wurde dabei der lößfreie, steilere westexponierte Hang unterschritten, auf dem am Ende der letzten Kaltzeit nur der Deckschutt (SEMmel 1964) vorhanden war (8 auf Abb. 4).

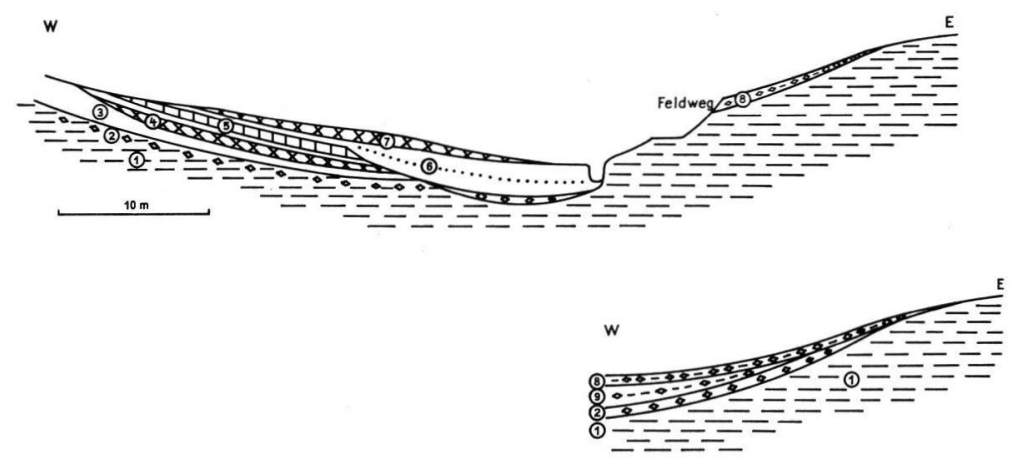

Abb. 4. Delle bei Frickenhausen (R 357954 / H 550554)

$1=$ anstehender Keuper; $2=$ Keuper-Schutt; $3=$ Riß-Löß; $4=$ Parabraunerde der letzten Warmzeit; $5=$ Altwürm-Humuszone; $6=$ Naßboden $\mathrm{E}_{2} ; 7=$ Parabraunerde; $8=$ Deckschutt mit Braunerde; $9=$ lößlehmhaltiger Keuper-Schutt.

In jüngerer Zeit sorgte die Bodenerosion für eine weitere Differenzierung der Form. Auffallenderweise wurde dabei im Dellentiefsten ebenfalls stark abgetragen. Ahnliche Beobachtungen sind schon mehrfach mitgeteilt worden (HARD 1967, RICHTER 1965, Rohdenburg 1965, Semmel 1961, 1968). Dellenabwärts verflacht der westexponierte Hang etwas. Hier ist eine stärker differenzierte Schuttdeckenabfolge zu beobachten. $\mathrm{Zu}-$ unterst liegt über dem anstehenden Lettenkeuper ein reiner Keuperschutt (2), der dem „Basisschutt“ entspricht. Darüber folgt der lößlehmhaltige „Mittelschutt“ (9), der hangaufwärts auskeilt. Das Hangende bildet schließlich der Deckschutt (8), der weniger Lößlehm und mehr Keuperbrocken enthält. Im höchsten Hangbereich liegt dieser Schutt direkt auf dem anstehenden Keuper. Junge Bodenerosion hat den Deckschutt am oberen Hang abgetragen. 


\subsection{Aufgefül1te Delle bei Eibelstadt}

Im Gegensatz zu der eben beschriebenen Form gibt es - wie auch schon aus dem Rhein-Main-Gebiet berichtet (Semmel 1968, 116) - Dellen, die im Jungwürm vollkommen aufgefüllt wurden und heute morphographisch nicht mehr in Erscheinung treten. Als Beispiel aus Franken sei auf den Steinbruch verwiesen, der westlich des Eichenhölzle zwischen Sommerhausen und Eibenstadt liegt (R 357380/H 550940). In ihm befindet sich eine Steinschleiferei. Hier ist inmitten der Quaderkalke des Oberen Muschelkalks eine Delle eingetieft, die umgelagerte Reste des letztwarmzeitlichen Bodens sowie drei Altwürm-Humuszonen enthält. Darüber folgt oberer Jungwürmlöß mit dem Naßboden $\mathrm{E}_{2}$. Die Delle war bereits am Ende des Würms völlig eingedeckt. Heute beginnt an der Oberfläche erst weiter östlich eine Delle. Auch hier fand also eine Verlegung der Hohlform nach Osten statt.

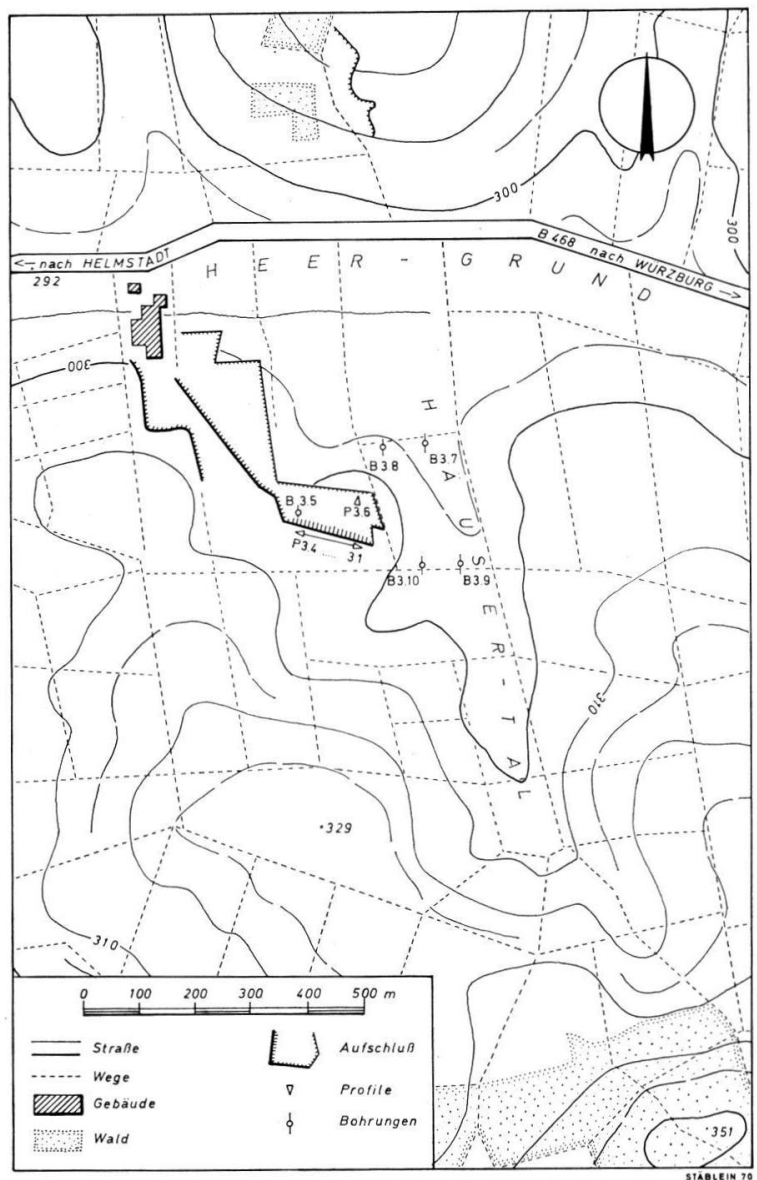

Abb. 5. Trockental-System des Heergrundes östlich Helmstadt mit Lage der Profile und Bohrpunkte.

\section{Das Häuser-Tal bei Helmstadt westlich Würzburg}

Während die Entwicklung der bisher beschriebenen Formen erst ab der vorletzten Kaltzeit zu verfolgen ist, erlauben die Aufschlußverhältnisse in der östlichen Umgebung von Helmstadt einen Einblick in wesentlich ältere Formungsstadien des dortigen Häuser- 
Tales. Dieses Tal ist ein Trockental, das östlich von Helmstadt in das große TrockentalSystem des Heer-Grundes einmündet (Abb.5) und von Rücken flankiert wird, die in ihren Kernen aus Muschelkalk bestehen. In der westlichen Flanke des Häuser-Tales liegt die Lehmgrube der Ziegelei Helmstadt. Dort sind vier fossile, teilweise pseudovergleyte Parabraunerden aufgeschlossen (Abb. 6). Sie fallen sämtlich sehr gleichmäßig nach ESE ein. Während unter dem ältesten fossilen $\mathrm{B}_{\mathrm{t}}$-Horizont noch über $5 \mathrm{~m}$ kalkfreier Löß liegen, ist $\mathrm{zwischen} \mathrm{den} \mathrm{einzelnen} \mathrm{B}_{\mathrm{t}}$-Horizonten die Lößmächtigkeit gering. Auch dort gibt es keinen kalkhaltigen Löß mehr, allenfalls nur noch Lößkindl ( $\left.\mathrm{CaCO}_{3}-K o n k r e t i o n e n\right)$. Relativ gut und mächtig ist dagegen im östlichen Grubenbereich der Würmlöß entwickelt. Dort kommt kalkhaltiger Löß sowohl unter als auch über dem Lohner Boden vor.

Aus geomorphologischer Sicht fällt sofort die allmähliche Wanderung des Hanges in östlicher Richtung auf. Der auf Abb. 6 als sicher nachgewiesen dargestellte Schichtenverlauf zeigt, daß das Maß der Eintiefung während der Verlegung sehr gering war. Es erreicht noch nicht einmal $2 \mathrm{~m}$ ! Dabei muß selbstverständlich berücksichtigt werden, wieviel Zeit zur Verfügung stand.

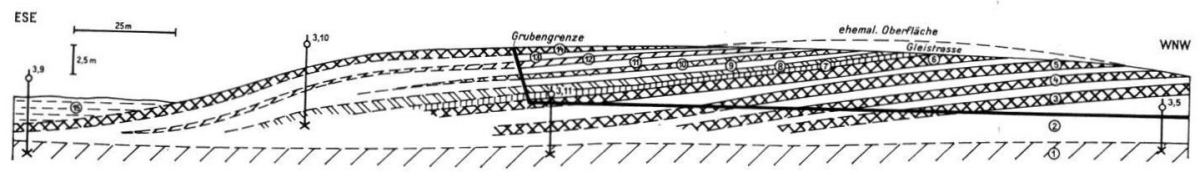

Abb. 6. Lehmgrube der Ziegelei Helmstadt und angrenzendes Häuser-Tal.

$1=$ Muschelkalk-Mergel mit Verwitterungs- und Schuttdecke; $2=$ ältester Lößlehm; $3=$ erster fossiler $\mathrm{B}_{\mathrm{t}}$-Horizont; $4=\mathrm{zw}$ eiter fossiler $\mathrm{B}_{\mathrm{t}}$-Horizont; $5=$ dritter fossiler $\mathrm{B}_{t}$-Horizont; $6=$ vierter fossiler $\mathrm{B}_{\mathrm{t}}$-Horizont mit ${ } \mathrm{A}_{\mathrm{l}}$-Horizont"; $7=$ Altwürm-Humuszone; $8=$ Umlagerungszone; $9=$ Mittelwürm-Löß; $10=$ Lohner Boden; $11=$ Jungwürm-Löß (älterer); $12=\mathrm{Naß-}$ boden $\mathrm{E}_{2} ; 13=$ Jungwürm-Löß (jüngerer); $14=$ Parabraunerde; $15=$ Kolluvium. 3,5; 3,9; 3,$10 ; 3,11=$ Bohrungen.

Leider stellt sich hier einmal mehr die Frage nach dem stratigraphischen und - damit verbunden - klimatischen Wert der fossilen Parabraunerden (SEMmeL 1967). Wenn man neuere Untersuchungsergebnisse aus dem östlichen Mitteleuropa (KukLA 1969, Tab. 32) zugrundelegen darf, so erfolgte die Bildung in tensiver Parabraunerden in Mitteleuropa im mittleren und jüngeren Pleistozän nur während echter Warmzeiten. Solche Warmzeiten hat es aller Wahrscheinlichkeit nach erheblich mehr gegeben, als gemeinhin bisher angenommen wurde. Daß auch im mittleren Pleistozän, also etwa in dem bisher als Saale-Kaltzeit bezeichneten Abschnitt, intensive Parabraunerden entstanden, scheinen die von PAAs (1968) mitgeteilten Befunde aus dem niederrheinischen Lößgebiet zu bestätigen. Dort liegen auf den Kiesen der Unteren Mittelterrasse, die in den Drenthe-Abschnitt der Saalezeit eingestuft werden, Lösse mit insgesamt drei fossilen, intensiven Parabraunerden. Wenn die stratigraphische Einordnung der liegenden Terrassenkiese zutrifft, ist nicht daran zu zweifeln, daß vor der eemzeitlichen Parabraunerde schon zwei ältere Böden dieses Typs, die in die Saalezeit gehören, gebildet wurden.

Sollte das auch im Helmstädter Proenl zutreffen, so wäre dennoch der dortige älteste $\mathrm{B}_{\mathrm{t}}$-Horizont immer noch als prä-saalezeitlich und der Löß darunter als Produkt der ElsterKaltzeit anzusehen. Will man Brunnacker $(1964,77)$ folgen und jeden fossilen $B_{t}$-Horizont einem Interglazial der klassischen süddeutschen Pleistozän-Gliederung zuordnen, so wäre der älteste $B_{t}$-Horizont sogar als Prä-Günz zu datieren. Wobei selbstverständlich auch hier die Frage offenbleibt, wie norddeutsche und süddeutsche Glazialstratigraphie $\mathrm{zu}$ parallelisieren sind. Unabhängig davon läßt sich aber zeigen, daß das Helmstädter Profil einen langen Zeitraum für seine Entwicklung zur Verfügung gehabt hat, der nach gegenwärtig üblicher Gliederung in das ältere Pleistozän zurückreicht. 
Während dieser Zeit wanderte das Trockental oder zumindest sein Westhang und sein Zentrum immer weiter nach ESE, ohne sich dabei in nennenswertem Maße in den liegenden Mittleren Muschelkalk stärker einzutiefen. Die Hauptabtragung wurde am Osthang (westexponiert) geleistet. Hier tritt heute der Muschelkalk häufig zutage oder die Lößbedeckung beträgt meist nur 1-2 m Stärke. Durch die junge Bodenerosion wurde hier und stellenweise auch auf dem Gegenhang die Klimax-Parabraunerde teilweise oder vollständig abgetragen. Die abgeschwemmten Massen füllten die Hohlform zum Teil wieder auf, so daß dort gegenwärtig erheblich mehr Lockermaterial liegt als am Ende des Pleistozäns. $\mathrm{Zu}$ jener Zeit lagerte im Dellentiefsten nur ca. $1 \mathrm{~m}$ Löß, der von der späteren Bodenbildung vollkommen erfaßt wurde. Auch der liegende mm-Mergel mit einer geringmächtigen Schuttlage ist oberflächlich noch verwittert.

Der Bereich der westlich anschließenden mächtigen Lößdecke wurde im jüngeren Pleistozän durch eine neu eingeschnittene Delle differenziert, die nördlich der Bohrung $3.5^{1}$ ) verläuft. Die Delle schneidet die fossilen $B_{t}$-Horizonte. In dem Rücken, der zwischen ihr und dem Häuser-Tal liegt, sind mindestens noch drei fossile $\mathrm{B}_{\mathrm{t}}$-Horizonte erhalten. Aus technischen Gründen konnte der liegende Muschelkalk in der dort angesetzten Bohrung B 3.8 nicht erreicht werden. Die Abfolge stimmt weitgehend mit dem ebenfalls im zentralen Teil des Rückens aufgenommenen Profil P 3.6 (an der Nordwand der Ziegeleigrube) überein. Die östlich von B 3.8 im tiefsten Teil des Häuser-Tals liegende Bohrung B 3.7 enthält dagegen keinen pleistozänen $\mathrm{B}_{\mathrm{t}}$-Horizont mehr. Unter 1,5 $\mathrm{m}$ Kolluviallehm folgt die holozäne Parabraunerde. Sie wird von $0,9 \mathrm{~m}$ kalkhaltigem Löß unterlagert, unter dem sich geringe Mengen von Lößlehm und tonigem Muschelkalkschutt befinden. Im Lößlehm sind umgelagerte Reste von Humuszonen und basal $10 \mathrm{~cm} \mathrm{fB}$-Material anzutreffen. Der $\mathrm{fB}_{\mathrm{t}}$-Rest scheint in situ zu liegen. Demnach ist hier vermutlich schon zu Beginn der letzten Kaltzeit das Häuser-Tal bis in diese Tiefe ausgebildet gewesen.

Zusammenfassend ist festzustellen, daß im Bereich des Häuser-Tales im älteren Pleistozän der Obere und Mittlere Muschelkalk bis in das Niveau des heutigen "Talbodens“ zerschnitten waren. Von damals an - vielleicht auch schon früher — setzte eine Wanderung der Haupthohlform nach Osten (genauer ESE) ein. Auf dem ostexponierten Hang wurden Lösse sedimentiert mit einer Gesamtmächtigkeit von mindestens $8 \mathrm{~m}$. Dabei bildeten sich in klimatisch günstigen Perioden Böden mit warmzeitlichen Habitus. Die Gründe für die teilweise recht unterschiedliche Lößmächtigkeit zwischen den einzelnen Böden sind nicht sicher bekannt.

Durch Anlage von Sekundär-Dellen wurde die Lößdecke zerschnitten. Auf diese Weise entstand im Muschelkalkgebiet ein Relief, das völlig aus Löß aufgebaut ist. Im Bereich der wandernden Tiefenlinien der Hohlformen blieb die Ausräumung fast immer stärker als die Lößakkumulation. Erst im jüngeren Holozän wurden diese Teile der Hohlformen durch Abschwemm-Massen, die im Gefolge der Bodenerosion entstanden, teilweise wieder aufgefüllt.

Gegen diese Darstellung des Entwicklungsganges könnte eingewendet werden, es habe bereits zu Beginn der Lößakkumulation eine - vielleicht noch präquartäre - Form bestanden, die allmählich von Westen nach Osten mit Löß aufgefüllt wurde. Reste von Rotlehmen, die zur Stützung dieser These dienen könnten, sind jedoch nicht gefunden worden. Wichtiger aber scheint uns, daß bei den Aufschlüssen in der Kitzinger Umgebung deutlich zu erkennen ist, daß die Hohlformen schrittweise in das Anstehende in Richtung Osten eingeschnitten werden. Es ist wohl anzunehmen, daß sich die Entwicklung im Häuser-Tal ähnlich vollzogen hat.

1) Bei den Bohrarbeiten waren uns freundlicherweise die Herren Bahlke, Claus, Fugel und Späth vom Geographischen Institut Würzburg behilflich. 
Abschließend sei noch auf einen Talanfang bei Bamberg verwiesen, der eine von Westen nach Osten fortschreitende Auffüllung zeigt. Es handelt sich dabei um ein Profil, das durch die Grube der Gaustädter Ziegelwerke aufgeschlossen ist. ${ }^{2}$ ) Dort sind KeuperSolifluktionsmassen mit Lößeinschaltungen im oberen Bereich zu beobachten, die einen von W nach E ziehenden Talanfang vollkommen ausfüllen. In den Solifluktionsmassen sind vier fossile Böden vom Warmzeit-Typus (Parabraunerden bzw. Pseudogley-Parabraunerden) ausgebildet, die die einzelnen Stadien der von W nach E erfolgenden Auffüllung sehr gut dokumentieren und zugleich die altpleistozäne Anlage der Hohlform beweisen. Allerdings scheint im Bereich der heutigen Hohlform die jüngere Eintiefung weit unter die altpleistozäne hinabzureichen. Weshalb dies in anderen Fällen so häufig nicht zutrifft, soll an anderer Stelle im Zusammenhang mit Beobachtungen zur quartären Eintiefung der Flüsse diskutiert werden.

2) Die heutige Grube entspricht nicht den von BrunNacker (1956) und HöHL (1958) untersuchten Profilen in Gaustadt. Eine ausführliche Beschreibung des jetzigen Gaustädter Profils und anderer lößarmer bzw. lößfreier Quartär-Profile von geomorphologischer und stratigraphischer Bedeutung aus diesem Gebiet ist gemeinsam mit H. SРётH vom Geographischen Institut der Univversität Würzburg in Vorbereitung.

\section{Literatur}

Bartels, G. \& Rohdenburg, H.: Fossile Böden und Eiskeilhorizonte in der Ziegeleigrube Breinum (Niedersächsisches Bergland) und ihre Auswertung für die Reliefentwicklung im Jungquartär. - Gött. bodenkundl. Ber., 6, Göttingen 1968.

BrunNACKER, K.: Das Lößprofil in Kitzingen (Unterfranken). - Germania, 34, 3-11, 3 Abb., 1 Tab., Berlin 1956.

- : Würmeiszeitlicher Löß und fossile Böden in Mainfranken. - Geol. Bavar., 25, 22-38, München 1956 (1956a).

- : Zur Parallelisierung des Jungpleistozäns in den Periglazialgebieten Bayerns und seiner östlichen Nachbarländer. — Geol. Jb., 76, 129-150, 5 Abb., 3 Tab., Hannover 1959.

- : Über Ablauf und Altersstellung altquartärer Verschüttungen im Maintal und nächst dem Donautal bei Regensburg. - Eiszeitalter u. Gegenwart, 15, 72-80, 1 Abb., 1 Tab., Öhringen 1964.

FräNZLE, O.: Zertalung und Hangbildung im Bereich der Süd-Ville. - Erdkde., 23, 1-9, 2 Abb., 1 Karte, Bonn 1969.

Hard, G.: Lößschleier, Waldrandstufe und Delle. - Decheniana, 118, 181-197, 10 Abb., 3 Taf., Bonn 1967.

HöHL, G.: Zur Frage der Entstehung des Gaustädter Profils (Franken). - Mitt. geogr. Ges. Wien, 100, 77-89, 4 Tab., 6 Beil., 4 Bild., Wien 1958.

KukLA, J.: Die zyklische Entwicklung und die absolute Datierung der Löß-Serien; in DeMEK, J. \& KukLA, J. (Redakteure): Periglazialzone, Löß und Paläolithikum der Tschechoslowakei. 1-156, 50 Abb., 12 Tab., Brno 1969.

PAAs, W.: Stratigraphische Gliederung des Niederrheinischen Lösses und seiner fossilen Böden. Decheniana, 121, 9-38, 10 Abb., 2 Tab., Bonn 1968.

Richter, G.: Bodenerosion, Schäden und gefährdete Gebiete in der Bundesrepublik Deutschland. - Forsch. deutsch. Landeskde., 152, 1-592, zahlr. Abb., Bad Godesberg 1965.

Rohdenburg, H.: Untersuchungen zur pleistozänen Formung am Beispiel der Westabdachung des Göttinger Waldes. - Gießener geogr. Schr., 7, 1-76, 23 Fig., 16 Bild., 2 Ktn., Gießen 1965.

- : Jungpleistozäne Hangforschung in Mitteleuropa - Beiträge zur Kenntnisse, Deutung und Bedeutung ihrer räumlichen und zeitlichen Differenzierung. - Gött. bodenkdl. Ber., 6, 3-107, zahlr. Abb., Göttingen 1968.

Rohdenburg, H. \& Meyer, B.: Zur Feinstratigraphie und Paläopedologie des Jungpleistozäns nach Untersuchungen an südniedersächsischen und nordhessischen Lößprofilen. - Mitt. deutsch. bodenkdl. Ges., 5, 1-135, zahlr. Abb., Göttingen 1966.

Schönhals, E., Rohdenburg, H. \& Semmel, A.: Ergebnisse neuerer Untersuchungen zur Würmlößgliederung in Hessen. - Eiszeitalter u. Gegenwart, 15, 199-206, 1 Abb., Ơhringen 1964.

Semmel, A.: Beobachtungen zur Genese von Dellen und Kerbtälchen im Löß. - Rhein-Main. Forsch., 50, 135-140, 2 Abb., Frankfurt a. M. 1961.

- : Junge Schuttdecken in hessischen Mittelgebirgen. - Notizbl. hess. L.Amt Bodenforsch., 92, 275-285, 3 Abb., 1 Tab., Wiesbaden 1964. 
Semmel, A.: Neue Fundstellen von vulkanischem Material in hessischen Lössen. - Notizbl. hess. L.Amt Bodenforsch., 95, 104-108, 1 Abb., Wiesbaden 1967.

- : Über Prä-Würm-Lösse in Hessen. - Notizbl. hess. L.Amt Bodenforsch., 95, 239-241, Wiesbaden 1967. (1967a).

- : Studien über den Verlauf jungpleistozäner Formung in Hessen. - Frankf. geogr. Hefte, 45, 1-133, 35 Abb., Frankfurt a. M. 1968.

- : Bemerkungen zur Würmlößgliederung im Rhein-Main-Gebiet. - Notizbl. hess. L.Amt Bodenforsch., 97, 395-399, 1 Abb., Wiesbaden 1969.

Semmel, A. \& Plass, W.: Spätkaltzeitliche Umlagerungen in Parabraunerde-Profilen. - Mitt. deutsch. bodenkdl. Ges., 4, 33—40, 1 Abb., Göttingen 1965.

Manuskript eingeg. 22. 10. 1970.

Anschriften der Verf.: Prof. Dr. Arno Semmel, Geographisches Institut der Johann-WolfgangGoethe-Universität, 6 Frankfurt am Main, Senckenberganlage 36; Priv.-Doz. Dr. Gerhard Stäblein, Geographisches Institut der Julius-Maximilians-Universität, 87 Würzburg, Klinikstraße 3 . 